\title{
Valor nutritivo de lanches consumidos por escolares de uma escola particular
}

\author{
Nutritional value of snacks eaten by private schoolchildren
}

\author{
Aline Veroneze de Mello ${ }^{a}$, Juliana Masami Morimotob, Ana Carolina Almada Colucci Paternez ${ }^{\mathrm{c}}$ \\ a Nutricionista graduada pela Universidade Presbiteriana Mackenzie. \\ b Nutricionista. Doutora em Ciências, área de concentração em Nutrição em Saúde Pública. Docente da Universidade Presbiteriana Mackenzie. \\ c Nutricionista. Doutora em Saúde Pública, área de concentração em Nutrição em Saúde Pública. Docente da Universidade Presbiteriana Mackenzie.
}

RESUMO Objetivo: Avaliar o valor nutritivo de lanches consumidos por escolares, no horário do intervalo, de uma escola privada de São Paulo.

Materiais e Métodos: A amostra foi composta por 52 alunos de ambos os sexos do 10 ano do ensino fundamental. Realizou-se a análise quantitativa da refeição em relação à quantidade de energia, carboidratos, fibra alimentar, proteínas, lipídeos, sódio, cálcio e ferro. Também foi realizada análise qualitativa de forma a identificar os grupos alimentares consumidos. Os dados de ingestão de energia e nutrientes foram comparados aos parâmetros para lanches do Programa Nacional de Alimentação Escolar (PNAE).

Resultados: Verificou-se que as crianças que compram os lanches na cantina da escola, optam por alimentos mais energéticos em comparação com as que levam de casa. Os principais grupos de alimentos encontrados nas lancheiras foram cereais, leite e derivados e sucos artificiais. Além disso, os valores encontrados através da ingestão de energia e dos macronutrientes estão acima do recomendado, com exceção de proteína para o sexo masculino que se encontra abaixo do valor recomendado. Já os valores de micronutrientes encontrados estão abaixo do recomendado para o cálcio e acima do recomendado para o ferro.

Conclusão: $\mathrm{O}$ valor nutritivo de lanches consumidos por escolares, no horário do intervalo, de uma escola privada de São Paulo, aponta para a ingestão de energia e dos macronutrientes acima das recomendações do PNAE, com exceção de proteína para o sexo masculino, consumo de cálcio abaixo do recomendado e consumo excessivo de ferro. Apesar do baixo desperdício e das poucas crianças que compravam os lanches na cantina da escola optando por alimentos mais energéticos em comparação com as que levavam de casa, os resultados demonstraram inadequações que alertam para a necessidade do incentivo e da implementação de programas de educação nutricional nas escolas para contribuir com melhorias na qualidade de vida e na alimentação das crianças.

Palavras-chave: escolares; valor nutritivo; lanches; hábitos alimentares. 


\section{INTRODUÇÃO}

A alimentação infantil deve ser boa tanto qualitativamente, quanto quantitativamente, pois deverá garantir o fornecimento de todos os nutrientes necessários para o bom funcionamento do organismo e para uma boa nutrição $0^{1,2}$. O estado nutricional das crianças representa uma influência decisiva nos riscos de morbimortalidade, no crescimento e no desenvolvimento infantil, o que permite que a avaliação nutricional torne-se um importante instrumento que, por meio de procedimentos diagnósticos, possibilitem precisar a dimensão, o comportamento e os determinantes dos agravos nutricionais, assim como identificar os grupos de risco e as intervenções nutricionais adequadas ${ }^{3}$.

A mudança na alimentação infantil se deu por conta do crescimento de novas modalidades de alimentos que facilitam o transporte, o armazenamento, o preparo e até mesmo o consumo. Além disso, através de propagandas, embalagens e rótulos atrativos, as crianças acabam sendo estimuladas a consumirem alimentos ricos em açúcares, sódio e gorduras e pobres em fibras, vitaminas e minerais ${ }^{4}$. A família representa a maior influência para as crianças que tendem a imitar os hábitos alimentares dos pais. As escolhas de aquisição, de preparo e de consumo dos alimentos são incorporadas aos comportamentos das crianças ${ }^{5}$.

As crianças passam grande parte do dia nas escolas e consomem lanches no intervalo. Para isso, existe a necessidade do preparo de lanches em casa por sua família ou, às vezes, as crianças compram os lanches na cantina da própria escola. Dessa forma, uma análise para adequação dos produtos alimentares consumidos neste local deve ser realizada com a finalidade de melhorias na sua qualidade nutricional. Contudo, as escolas devem promover ações que estimulem as crianças a terem uma alimentação saudável e equilibrada, já que o ambiente escolar tem um papel fundamental para a formação dos hábitos alimentares ${ }^{6,7,8,9}$.

As relações entre os hábitos alimentares incorporados em casa e os aprendidos na escola podem interagir e um acabará influenciando o outro. Uma alternativa para tentar reduzir os casos de sobrepeso e obesidade entre crianças são programas de educação nutricional que irão garantir informações corretas sobre alimentação e, consequentemente, criarão um ambiente favorável à saúde e à promoção de hábitos alimentares saudáveis ${ }^{10}$.

Com isso, as mudanças no estilo de vida e nos hábitos e padrões alimentares, principalmente pelo aumento do consumo de alimentos ricos em açúcar e gordura de baixo custo e maior acessibilidade, representados pelo consumo de guloseimas (como bolachas recheadas, salgadinhos, doces) e refrigerantes, tem feito com que a prevalência da obesidade em crianças e adolescentes tenha aumentado em vários países do mundo, inclusive no Brasil ${ }^{11}$. Por isso, o consumo alimentar tem sido relacionado à obesidade não somente quanto ao volume da ingestão alimentar, como também à composição e qualidade da dieta ${ }^{12}$.

O PNAE (Programa Nacional de Alimentação Escolar), criado em 1955, tem por objetivo fornecer alimentação nas escolas para atender as necessidades dos alunos de creches e da educação básica durante o período em que estão na aula. Isso irá garantir a formação de hábitos alimentares saudáveis, assim como a contribuição para o desenvolvimento e rendimento escolar das crianças e dos jovens ${ }^{13}$.

O objetivo principal do presente estudo foi avaliar o valor nutritivo de lanches consumidos por escolares no horário do intervalo de uma escola privada do município de São Paulo. Entre os objetivos específicos, pretendeu-se avaliar a proporção de crianças que compram o lanche na escola e aquelas que levam de casa; avaliar diferenças entre os alimentos levados de casa e os realmente consumidos.

\section{MATERIAIS E MÉTODOS}

O estudo de delineamento transversal foi realizado em uma escola privada da região metropolitana de São Paulo de agosto de 2013 a julho de 2014. A amostra foi composta por alunos de ambos os sexos do ensino fundamental matriculados no 10 ano da escola privada escolhida. Foram considerados tanto os alunos que levam o lanche de casa quanto àqueles que compram na cantina ou na lanchonete da escola.

A pesquisa foi realizada pela própria pesquisadora, no mês de março de 2013, que avaliou o conteúdo das lancheiras das crianças e os alimentos consumidos antes da hora do intervalo. A coleta de dados se iniciou na sala de aula, cerca de 20 minutos antes do horário do intervalo. Neste momento, a pesquisadora entrou na sala de aula e por meio de um formulário de coleta de dados previamente elaborado, identificou os alunos que trouxeram lanche de casa, anotando a idade, gênero, o responsável pela preparação do lanche, os alimentos trazidos e as respectivas quantidades. Os alunos que compraram seu lanche na cantina da escola foram acompanhados pela pesquisadora para que fossem anotadas as informações sobre gênero, idade, o responsável por fornecer dinheiro para a compra do lanche e os alimentos comprados (com suas respectivas quantidades).

Ao final do horário do intervalo, a pesquisadora retornou para sala de aula para verificar se tudo o que foi trazido de casa foi consumido; em caso negativo, foi anotado tudo o que não foi consumido para descontar das informações inicialmente coletadas. Todos os escolares também foram 
perguntados sobre quem foi a pessoa que montou a lancheira e se alguém da família orientou sobre o que deveria ser consumido no horário do intervalo.

Posteriormente, realizou-se a análise quantitativa da refeição, por meio do cálculo do valor nutritivo em relação à quantidade de energia, carboidratos, fibra alimentar, proteínas, lipídeos, sódio, cálcio e ferro, com auxílio do software Avanutri Online ${ }^{\circledR}$. Também foi realizada análise qualitativa de forma a identificar os grupos alimentares consumidos: cereais, bolos e biscoitos, doces, frutas, sucos artificiais, frios e embutidos, óleos e gorduras, leite e derivados.

Os dados de ingestão de energia e nutrientes foram comparados aos parâmetros para lanches do Programa Nacional de Alimentação Escolar (PNAE). Para esta comparação foram calculados a média e o desvio padrão da ingestão de energia e nutrientes, segundo gênero. Os dados foram tabulados no programa Microsoft Excel 2010.

Por se tratar de uma pesquisa com seres humanos o projeto foi aprovado pelo Comitê de Ética em Pesquisa (CEP- CAAE no 0074.0.272.000-10) da Universidade Presbiteriana Mackenzie. O estudo foi conduzido após a autorização do responsável pela escola, por meio da assinatura do Termo de Consentimento Livre e Esclarecido à Instituição, assim como a autorização dos pais dos escolares com a assinatura do Termo de Consentimento Livre e Esclarecido aos Sujeitos de Pesquisa. Todos os procedimentos da pesquisa respeitaram as diretrizes da atual legislação sobre ética em pesquisa com seres humanos.

\section{RESULTADOS}

A amostra foi constituída por 52 escolares de ambos os sexos matriculados no $1^{\circ}$ ano do ensino fundamental de uma escola privada que permaneciam apenas meio período na escola (perído matutino ou vespertino), sendo que destes, $51,92 \%(n=27)$ eram do sexo masculino e $48,08 \%(n=25)$ eram do sexo feminino, com idades entre 5 e 7 anos. A média de idade foi de 5,8 $\pm 0,2$ anos. Os que relataram que a preparação do lanche foi feita pela mãe representam $86,54 \%$ (Tabela 1).

A Figura 1 representa a proporção de alunos que trouxeram determinados grupos de alimentos nas lancheiras no dia de coleta. No total, foram identificados 186 variedades de alimentos trazidos de casa, sendo que a maior parte das crianças trouxe cereais (67\%), leite e derivados (63\%) e sucos artificiais (62\%). Os resultados obtidos também apontam que os escolares consomem poucos alimentos dos seguintes grupos no horário do intervalo: óleos e gorduras (1\%), frios e embutidos (19\%) e doces (25\%).

De maneira geral, as crianças não desperdiçam os alimentos e a maior parte consome todo lanche que é
Tabela 1. Distribuição das características de escolares e dos lanches $(n=52)$.

\begin{tabular}{lcc}
\hline \multicolumn{1}{c}{ Características } & n & $\%$ \\
Sexo & & \\
$\quad$ Masculino & 27 & 48,08 \\
Feminino & 25 & 51,92 \\
Idade (anos) & & \\
5 & 12 & 23,08 \\
6 & 38 & 73,08 \\
7 & 2 & 3,84 \\
Preparação do lanche & & \\
Mãe & 45 & 86,54 \\
Pai & 3 & 5,76 \\
Auxiliar doméstica & 2 & 3,85 \\
Compra na escola & 2 & 3,85 \\
Elaboração do lanche & & \\
Casa & & \\
Lanchonete da escola & 50 & 96,15 \\
\hline
\end{tabular}

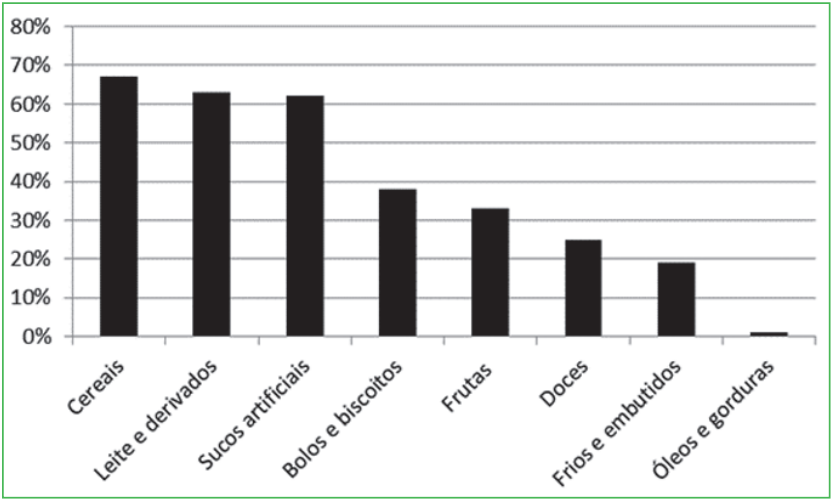

Figura 1. Distribuição de escolares segundo grupos de alimentos consumidos no horário do intervalo $(n=52)$.

levado de casa ou que é comprado na cantina da escola. De todos os alimentos trazidos, 18 não foram consumidos e 20 foram levados, mas não foram totalmente consumidos. O grupo de alimentos que as crianças mais desperdiçaram deixando de consumir totalmente, sendo levado de casa, foi o das frutas $(4,84 \%)$, seguido de cereais, sucos artificiais e bolos e biscoitos, cada um com 1,61\% de crianças que não consumiram os alimentos. Já entre os alimentos levados de casa e parcialmente consumidos estão os sucos artificiais $(4,30 \%)$, seguido de cereais $(2,15 \%)$ e bolos e biscoitos $(1,61 \%)$. Todos estes resultados representaram valores percentuais baixos, o que demonstrou baixo desperdício de alimentos.

O total de crianças que trouxeram lanche de casa representam 96,15\%, enquanto que apenas 3,85\% compraram o lanche na cantina da escola. Os alimentos mais comprados foram pão de queijo e suco artificial e os mais 
encontrados nas lancheiras foram bisnaguinha, iogurte de frutas, suco de fruta a base de soja, bolos recheados, maçã e chocolate (Figura 2). As duas crianças que compraram o lanche na cantina da escola foram acompanhadas até o local e notou-se que os lanches comprados apresentavam uma média de valor energético de 694,5 \pm 10 Kcal enquanto que a média de ambos os sexos que levaram o lanche de casa foi de $345,6 \pm 68,9 \mathrm{Kcal}$, o sexo feminino foi de $312,8 \pm 73,85 \mathrm{Kcal}$ e o sexo masculino $379,14 \pm 89,96 \mathrm{Kcal}$, mostrando-se elevado.

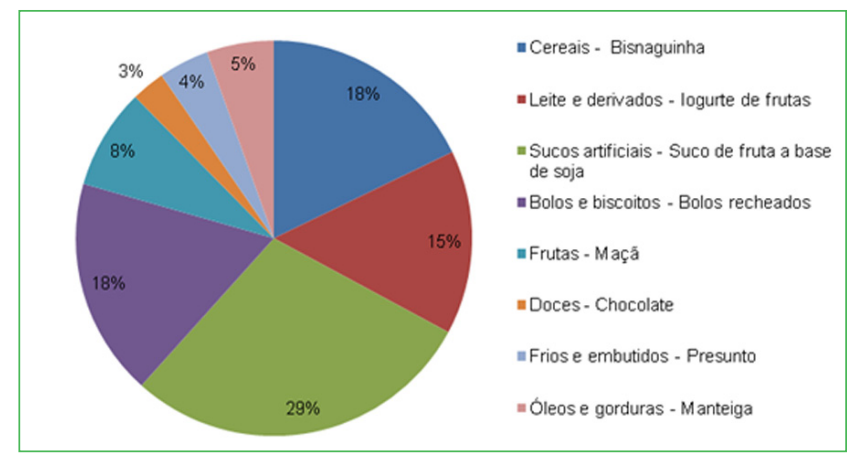

Figura 2. Distribuição em percentual dos alimentos mais levados segundo o grupo do alimento $(n=52)$.

A Tabela 2 apresenta a descrição do consumo de macronutrientes (carboidrato, fibra alimentar, proteína e lipídeo) e micronutrientes (ferro, sódio e cálcio) consumidos pelas crianças no horário do lanche. Ainda, pode-se perceber que os lanches apresentaram valor energético, lipídeo e carboidrato acima do recomendado pelo PNAE.

Dentre as crianças que participaram do estudo $11,53 \%$ levaram água em garrafinhas para o consumo durante $\mathrm{o}$ período de aula.

Tabela 2. Consumo de macronutrientes e micronutrientes segundo sexo e valores de recomendação estabelecidos pelo PNAE $(n=52)$.

\begin{tabular}{|c|c|c|c|}
\hline \multirow{2}{*}{ Nutriente } & \multicolumn{2}{|c|}{ Sexo } & \multirow{2}{*}{$\begin{array}{c}\text { Recomendação } \\
\text { PNAE } 20 \%\end{array}$} \\
\hline & Feminino & Masculino & \\
\hline Energia (Kcal) & $364,56 \pm 73,85$ & $379,14 \pm 89,96$ & 300 \\
\hline Carboidrato (g) & $57,63 \pm 12,86$ & $59,16 \pm 12,77$ & 48,8 \\
\hline Proteína (g) & $13,78 \pm 12,1$ & $7,71 \pm 2,08$ & 9,4 \\
\hline Lipídeo (g) & $11,33 \pm 3,61$ & $10,88 \pm 3,92$ & 7,5 \\
\hline Fibra alimentar (g) & $2,45 \pm 1,25$ & $3,45 \pm 1,69$ & 5,4 \\
\hline Ferro (mg) & $3,78 \pm 2,4$ & $5,1 \pm 3,31$ & 1,8 \\
\hline Sódio (mg) & $219,26 \pm 132,45$ & $221,38 \pm 100,71$ & - \\
\hline Cálcio (mg) & $195,35 \pm 32,2$ & $136,54 \pm 69,43$ & 210 \\
\hline
\end{tabular}

* Valores em média e desvio padrão. ** Fonte (Recomendação PNAE 20\%): Energia Organização das Nações Unidas para Agricultura e Alimentação (FAO), 2001; Carboidrato, Proteína e Lipídio - Organização Mundial de Saúde (OMS), 2003; Fibras, Vitaminas e Minerais - Referência da Ingestão Dietética (DRI) / Instituto de Medicina Americano (IOM), 1997 - 2000 - 2001. Adaptada para Resolução n³ 38/2009 do FNDE.

\section{DISCUSSÃO}

O presente estudo analisou a composição do lanche consumido por 52 escolares de uma instituição de ensino privada do município de São Paulo e comparou os resultados com os parâmetros de recomendação do PNAE. Apesar do baixo desperdício, os resultados demonstraram algumas inadequações que alertam para a necessidade constante de educação nutricional das crianças.

Matuk et al. ${ }^{14}$, em estudo realizado com crianças de ambos os sexos e idades entre 7 e 13 anos em cinco unidades de uma rede particular de ensino de São Paulo, demonstraram que $82 \%$ trouxeram cereais, $67 \%$ trouxeram sucos artificiais e outras bebidas e $65 \%$ trouxeram leite e derivados. Esses achados estão em conformidade com os resultados do presente estudo, o que pode indicar que normalmente os alimentos mais encontrados nas lancheiras são pertencentes a estes três grupos de alimentos. $\mathrm{O}$ alto consumo de refrigerantes e sucos artificiais foi observado em uma pesquisa realizada pelo Ministério da Saúde ${ }^{15}$ por inquérito telefônico, no qual houve um aumento de $13,4 \%$, em comparação ao ano de 2008, no número de consumidores de refrigerantes e sucos artificiais no Brasil, dado preocupante para a saúde pública no nosso país. Somente $11,53 \%$ das crianças levaram garrafinhas de água para o consumo, porém a instituição de ensino incentiva o consumo de água por meio da disponibilização de água em bebedouros localizados em diversos pontos de circulação da escola.

É importante ressaltar que a Portaria Interministerial no 1010, que instituiu diretrizes para a Promoção da Alimentação Saudável nas Escolas de educação infantil, fundamental e nível médio das redes públicas e privadas em âmbito nacional, incentiva o consumo de alimentos saudáveis como as frutas e considera que os refrigerantes e sucos artificiais não são considerados alimentos saudáveis ${ }^{16}$. O incentivo ao consumo alimentar adequado deve ser feito por meio de programas de educação alimentar e nutricional, como demonstrado no estudo de Deminice et al. ${ }^{17}$, realizado com alunos de uma escola municipal de Ribeirão Preto e com idades entre 6 e 16 anos, que teve como resultado principal que o programa de educação alimentar proposto foi eficaz na melhora do nível de conhecimento em alimentação e nutrição, o que aumenta a importância desse tipo de programa e mostra-se semelhante com os resultados encontrados por outros estudos realizados em escolares ${ }^{18}$. É importante ressaltar que a escola incentiva e tem projetos de educação nutricional no qual as crianças aprendem sobre alimentação e nutrição.

Estudos recentes demonstram que os alimentos das cantinas escolares são muito energéticos, ricos em açúcares, 
gorduras e sal, e indicam a preferência dos estudantes pelos mesmos $^{19,20}$. Porém, os pais podem intervir e influenciar as mudanças de hábitos alimentares e a atividade física de seus filhos, principalmente antes dos 10 anos, o que contribui para a redução de obesidade em crianças e pode proporcionar um auxílio nas melhores escolhas alimentares ${ }^{21}$.

De acordo com o Programa Nacional de Alimentação Escolar $^{22}$, os valores encontrados de ingestão de energia e dos macronutrientes estavam acima do recomendado, com exceção de proteína para o sexo masculino que se encontrou abaixo do valor recomendado que é de 9,4g para crianças do ensino fundamental entre 6 e 10 anos de idade. Estudo semelhante que avaliou o perfil qualitativo de lanches escolares de alunos matriculados nas $3^{\underline{a}}$ e $4 \stackrel{\text { a }}{\text { séries de }}$ uma escola particular no Distrito Federal identificou que os lanches trazidos de casa continham alimentos com elevada concentração de gordura e açúcar ${ }^{23}$, resultados semelhantes ao do presente estudo.

O consumo alimentar de crianças em idade escolar deve ser equilibrado tanto em quantidade quanto em qualidade. Isso é importante para o desenvolvimento cognitivo, melhor aprendizado e redução dos transtornos causados por deficiências de macro e micronutrientes, tais como desnutrição e anemia, além de evitar o surgimento precoce de doenças crônicas não transmissíveis ${ }^{24}$. A proteína é um macronutriente que possui diversas funções, destacando-se o fornecimento de aminoácidos essenciais e nitrogênio para a síntese de enzimas, hormônios, neurotransmissores, proteínas corporais e também para a manutenção e formação de tecidos. Com isso, o consumo proteico abaixo do ideal pode acarretar diversos prejuízos nas funções realizadas pelos aminoácidos. Por outro lado, o excesso no consumo de proteínas, em idades precoces, além de elevar a carga renal, está também relacionado com o risco de desenvolvimento de obesidade quando a criança se tornar um adulto ${ }^{25}$. Por estes motivos, a ingestão abaixo da recomendação observada no presente estudo entre os meninos é preocupante.

Os valores de micronutrientes encontrados no presente estudo estavam abaixo do recomendado pelo PNAE para o cálcio e acima do recomendado para o ferro. A tabela de referência do PNAE não apresenta valores de recomendação para o sódio. O ferro é o componente de enzimas que participam no processo de respiração celular, de maneira que se torna um mineral imprescindível no transporte de oxigênio e gás carbônico. Quando o consumo é inadequado, pode-se ter como consequência a deficiência que é denominada anemia, o que leva a prejuízos de crescimento e desenvolvimento cognitivo, afetando o desempenho escolar ${ }^{26}$. Neste estudo, o consumo de ferro foi acima do recomendado possivelmente pelo elevado consumo de cereais e também pelo consumo de bolos e biscoitos que contém em sua composição a farinha de trigo ou milho enriquecida com ferro ${ }^{27}$. O cálcio, por sua vez, é um mineral essencial ao organismo, e muito importante, uma vez que realiza funções como a participação na mineralização dos ossos, além de realizar a formação, manutenção da saúde e estrutura e rigidez óssea. Portanto, a baixa ingestão ou baixa absorção de cálcio em crianças e adolescentes pode limitar seu crescimento, sendo necessário fornecer quantidades suficientes de ambos nesta fase de desenvolvimento ${ }^{28}$.

O presente estudo demonstrou que as poucas crianças que compravam os lanches na cantina da escola optavam por alimentos mais energéticos em comparação com as que levavam de casa e, de modo geral, as crianças não desperdiçavam os alimentos. A maior parte das crianças trouxeram alimentos dos seguintes grupos de alimentos: cereais, leites e derivados e sucos artificiais. Com relação ao PNAE, os valores encontrados através da ingestão de energia e dos macronutrientes estão acima do recomendado, com exceção de proteína para o sexo masculino que se encontra abaixo do valor recomendado. Já os valores de micronutrientes encontrados estão abaixo do recomendado para o cálcio e acima do recomendado para o ferro.

Por fim, pode-se perceber que existe a necessidade do incentivo e da implementação de programas de educação nutricional nas escolas para contribuir com melhorias na qualidade de vida e na alimentação das crianças. Dessa forma, os pais também possuem fundamental importância na formação de hábitos alimentares saudáveis que serão mais eficazes se forem reforçadas pelas instituições de ensino.

\section{AGRADECIMENTOS}

A Universidade Presbiteriana Mackenzie que instituiu o Programa Institucional de Bolsas de Iniciação Científica (PIBIC) e ao CNPq pela bolsa de Iniciação Científica concedida para a realização da pesquisa.

\section{REFERÊNCIAS}

1. Spinelli MGN, Goulart RMM, Santos ALP, Gumiero LDC, Farhud CC, Freitas EB, Dantas LF. Consumo alimentar de crianças de 6 a 18 meses em creches. Rev Nutr. 2003;16(4):409-14. http://dx.doi. org/10.1590/S1415-52732003000400004

2. Philippi ST, Cruz ATR, Colucci ACA. Pirâmide alimentar para crianças de 2 a 3 anos. Rev Nutr. 2003;16(1):5-19. http://dx.doi. org/10.1590/S1415-52732003000100002

3. Castro TG, Novaes JF, Silva MR, Costa NMB, Franceschini SCC, Tinôco ALA, Leal PFG. Caracterização do consumo alimentar, ambiente socioeconômico e estado nutricional de pré-escolares de creches municipais. Rev Nutr. 2005;18(3):321-30. http://dx.doi. org/10.1590/S1415-52732005000300004 
4. Pontes TE, Costa TF, Marum ABFR, Brasil ALD, Taddei JAAC. Nutritional guidance for children and adolescents and the new consumption patterns: advertising, packaging and labeling. Rev Paul Pediatr. 2009;27(1):99-105. http://dx.doi.org/10.1590/S010305822009000100015

5. Gambardella AM, Frutuoso MF, Franch C. Prática alimentar de adolescentes. Rev Nutr. 1999;12(1):5-19. http://dx.doi.org/10.1590/ S1415-52731999000100005

6. Campos JADB, Zuanon ACC. Merenda escolar e promoção de saúde. Ciênc Odontol Bras. 2004;7(3):67-71. http://dx.doi. org/10.14295/bds.2004.v7i3.443

7. Ministério da Saúde (BR). Secretaria de Atenção à Saúde. Departamento de Atenção Básica. Brasília (DF); 2008 [acesso em 2014 set. 02]. Disponível em: http://bvsms.saude.gov.br/bvs/publicacoes/ manual_operacional_profissionais_saude $>$._educacao.pdf

8. Gonçalves FD, Catrib AMF, Vieira NFC, Vieira LJES. Health promotion in primary school. Interface Comun Saúde Educ. 2008;12(24): 181-92. http://dx.doi.org/10.1590/S1414-32832008000100014

9. Sociedade brasileira de pediatria. Obesidade na infância e adolescência: manual de orientação. São Paulo (SP): SBP; 2008 [acesso em 2015 jan. 14]. Disponível em: http://www.sbp.com.br/ PDFs/Man\%20Nutrologia_Obsidade.pdf

10. Buss PM. Promoção e educação em saúde no âmbito da Escola de Governo em Saúde da Escola Nacional de Saúde Pública. Cad Saúde Pública. 1999;15(2):177-85. http://dx.doi.org/10.1590/ S0102-311X1999000600018

11. Wang Y, Monteiro C, Popkin BM. Trends of obesity and underweight in older children and adolescents in the United States, Brazil, China and Russia. Am J Clin Nutr. 2002 Jun;75(6):971-7.

12. Triches RM, Giugliani ER. Obesidade, práticas alimentares e conhecimentos de nutrição em escolares. Rev Saúde Pública. 2005;39(4): 541-7. http://dx.doi.org/10.1590/S0034-89102005000400004

13. Ministério da Saúde (BR). Secretaria de Vigilância em Saúde. Departamento de Vigilância de Doenças e Agravos Não Transmissíveis e Promoção da Saúde. Vigitel Brasil - Saúde suplementar. Vigilância de fatores de risco e proteção para doenças crônicas por inquérito telefônico. Brasília (DF): Ministério da Saúde; 2013. p. 51-68.

14. Matuk TT, Stancari PCS, Bueno MB, Zaccarelli EM. Composição de lancheiras de alunos de escolas particulares de São Paulo. Rev Paul Pediatr. 2011;29(2):157-63. http://dx.doi.org/10.1590/S010305822011000200005

15. Ministério da Educação (BR). Fundação Nacional de Desenvolvimento da Educação (FNDE). Resolução/CD/FNDE n.38, de 16 de julho de 2009. Dispõe sobre o atendimento da alimentação escolar aos alunos da educação básica no Programa Nacional de Alimentação Escolar - PNAE. Brasília (DF): Ministério da Educação; 2009.

16. Ministério da Educação (BR). Fundação Nacional de Desenvolvimento da Educação (FNDE). Portaria Interministerial n.10, de 8 de maio de 2006. Institui as diretrizes para a Promoção da Alimentação Saudável nas Escolas de educação infantil, fundamental e nível médio das redes públicas e privadas, em âmbito nacional. Brasília (DF): Ministério da Educação; 2006.
17. Deminice R, Laus MF, Marins TM, Silveira SDO, Dutra-deOliveira JE. Impacto de um programa de educação alimentar sobre conhecimentos, práticas alimentares e estado nutricional de escolares. Alim Nutr. 2007;18(1):35-40.

18. Costa EQ, Ribeiro VMB, Ribeiro ECO. Programa de alimentação escolar: espaço de aprendizagem e produção de conhecimento. Rev Nutr. 2001;14(3):225-9. http://dx.doi.org/10.1590/S141552732001000300009

19. Schmitz BAS, Recine E, Cardoso GT, Silva JRM, Amorim NFA, Bernardon R, Rodrigues MLCF. A escola promovendo hábitos alimentares saudáveis: uma proposta metodológica de capacitação para educadores e donos de cantina escolar. Cad Saúde Pública. 2008;24(2):312-22. http://dx.doi.org/10.1590/S0102311X2008001400016

20. Menezes RCE, Osorio MM. Consumo energético-protéico e estado nutricional de crianças menores de cinco anos, no estado de Pernambuco, Brasil. Rev Nutr. 2007;20(4):337-47. http://dx.doi. org/10.1590/S1415-52732007000400001

21. Fernandes PS, Bernardo CO, Campos RMMB, Vasconcelos FAG. Avaliação do efeito da educação nutricional na prevalência de sobrepeso/obesidade e no consumo alimentar de escolares do ensino fundamental. J Pedriatr (Rio J). 2009;85(4):315-21. http:// dx.doi.org/10.2223/JPED.1917

22. Ministério da Educação (BR). Fundação Nacional de Desenvolvimento da Educação (FNDE). Programa Nacional de Alimentação Escolar - PNAE. Referências Nutricionais para o Programa Nacional de Alimentação Escolar. Brasília (DF): Ministério da Educação; 2009.

23. Mesquita JH, Pinto PCMM, Sarmento CTM. Perfil qualitativo dos lanches escolares consumidos em instituição de ensino particular do Distrito Federal - Brasil. Univ Ciênc Saúde. 2006;4(1): 49-62.

24. Abrantes MM, Lamounier JA, Colosimo EA. Prevalência de sobrepeso e obesidade em crianças e adolescentes das regióes Sudeste e Nordeste. J Pedriatr (Rio J). 2002;78(4):335-40. http:// dx.doi.org/10.1590/S0021-75572002000400014

25. Gunther AL, Remer T, Kroke A, Buyken AE. Early protein intake an later obesity risk: which protein sources at which time points throughout infancy and childhood are important for body mass index and body fat percentage at 7 years of age? Am J Clin Nutr. 2007;86(6):1765-72.

26. Cruz GF, Santos RS, Carvalho CMRG, Moita GC. Avaliação dietética em creches municipais de Teresina, Piauí, Brasil. Rev Nutr. 2001;14(1):21-32. http://dx.doi.org/10.1590/S141552732001000100004

27. Agência Nacional de Vigilância Sanitária (BR). Resolução RDC n.344, de 13 de dezembro de 2002. Aprova o Regulamento Técnico para a Fortificação das Farinhas de Trigo e das Farinhas de Milho com Ferro e Ácido Fólico. Brasília (DF): ANVISA; 2002.

28. Bueno AL, Czepielewski MA. A importância do consumo dietético de cálcio e vitamina D no crescimento. J Pedriatr (Rio J). 2008;84(5):386-94. http://dx.doi.org/10.2223/JPED.1816 